\title{
Movimientos sociales y estrategias de comunicación. El caso del 15-M y de Occupy Wall Street
}

\author{
Antonio CAstillo EsParcia \\ Universidad de Málaga \\ acastilloe@uma.es \\ Damián GARCÍA PONCE \\ Universidad de Málaga \\ damiangponce@hotmail.com \\ Emilia SMOLAK LOZANO \\ Universidad de Málaga \\ emilia.smolak@gmail.com
}

Recibido: $18 / 06 / 2012$

Aceptado: 17/01/2013

\section{Resumen}

El papel de los medios de comunicación es esencial en la manifestación y formulación de la opinión pública en las sociedades democráticas. Fruto es esa significación, los medios son receptores de múltiples influencias para conseguir presencia en su contenido redaccional y opinativo. Este artículo analiza la presencia de los movimientos antisistema en los casos de España (15-M) y de Estado Unidos (Occupy Wall Street) a través del análisis de los siete diarios españoles de mayor difusión y de su presencia en las redes sociales. De los resultados se desprende que el movimiento español se centró más en la acción, la movilización, una escasa profundidad analítica y las demandas políticas mientras que el movimiento estadounidense contó con mayor información cualitativa de expertos y un aspecto centrado en las demandas de reformas económicas.

Palabras clave: Comunicación política, estrategias de comunicación, periodismo, movimientos sociales

\section{Social Movements and Communication Strategies. The Case of 15-M and Occupy Wall Street}

\begin{abstract}
The role of mass media is essential to form and express the public opinion in the democratic societies. As the result of the significance of mass media in society is the fact that mass media are receptors of the multiple influences in an effort to achieve its presence in the journalistic content. This paper attempts to research the presence of anti-sistemic movements in the case of Spain (15-M) and United States (Occupy Wall Street). The methodology is based on the content analysis of seven Spanish newspapers and the presence of the movements in the online social networks. As the result of the investigation, it can be concluded that Spanish movement is mainly centered in action, mobilization and political demands, without contributing detailed and in-deep analysis of the system, whereas American movement was based on major expertise and qualitative information and on demands for economic reforms.

Keywords: Political communication, communication strategies, journalism, social movements.

\section{Referencia normalizada}

CASTILLO ESPARCIA, Antonio; GARCÍA PONCE, Damián; y SMOLAK LOZANO, Emilia (2013): "Movimientos sociales y estrategias de comunicación. El caso del 15-M y de Occupy Wall Street". Estudios sobre el mensaje periodístico. Vol. 19, Núm. 1, págs.: 71-89. Madrid, Servicio de Publicaciones de la Universidad Complutense.
\end{abstract}


Sumario: 1. Introducción. 2. Métodos. 3. Resultados; 3.1. Análisis de los diferentes diarios; 3.1.1. El País; 3.1.2. El Mundo; 3.1.3. ABC; 3.1.4. La Vanguardia; 3.1.5. La Razón; 3.1.6. El Periódico; 3.1.7. La Gaceta; 3.2. El tipo del contenido; 3.3. Fuentes de mensajes más utilizados; 3.4. El contenido del perfil: mensajes; 3.5. Occupy Wall Street. 4. Conclusión. 5. Referencias bibliográficas.

\section{Introducción}

En el sistema democrático influir sobre la opinión pública es hacerlo indirectamente sobre el poder político. Todo poder adiciona un grado de dificultad en su actividad cuando las medidas que pretende adoptar cuentan con una opinión pública desfavorable. Conocedores de esta situación, los grupos de presión realizan acciones sobre el público, como un ensayo de persuasión para desarrollar el reclutamiento o con la intención de crear y obtener un clima de simpatía y comprensión general acerca de sus demandas y aspiraciones ${ }^{1}$.

Uno de los principales poderes vertebradores entre sociedad y sistema político son los medios de comunicación. Tocqueville (1980: 174-175), en su análisis de la democracia en los Estados Unidos, afirma que los medios de comunicación permiten llegar hasta el más ínfimo lugar de la vida política, iluminando todos los resortes secretos de la política y forzando, de esta manera, a los hombres públicos a comparecer ante la opinión pública.

En este sentido, Milbraith (1963: 139) señala que los gobernantes elaboran una decisión a partir de lo que ellos perciben y no de lo que es objetiva u ontológicamente verdadero o real. Por lo tanto, una forma de influir sobre la decisión es incidir sobre las percepciones de los decisores públicos. La comunicación es, por lo tanto, para Milbraith un medio de cambiar o influir una percepción, esto es, el proceso del lobbying es un proceso de comunicación.

En el caso de los movimientos y organizaciones sociales se pretende crear la percepción de que el público simpatiza con sus aspiraciones y demandas presentadas o causas defendidas. Resulta difícil que, desde el poder público, se adopten decisiones contra un grupo que disfruta socialmente de una fuerte y generalizada corriente de opinión favorable.

Las campañas y las estrategias de comunicación de las organizaciones sociales, realizadas principalmente a través de los medios de comunicación, que se pueden ejecutar sobre el público han sido catalogadas por Finer (1966: 160-175) de dos maneras diferentes:

a) campañas en la base que pueden dirigirse a crear una imagen pública favorable al grupo o que permite establecer una exigencia pública concreta, para así incitar a los poderes públicos a adoptar decisiones sobre estas exigencias. En el

1 Existe una amplia bibliografía sobre el estudio de la opinión pública que el lector encontrará restringida, en este estudio, a aquellas investigaciones que han tenido una repercusión remarcable. Meynaud (1966: 413) cita ejemplos de acción sobre la opinión pública como son los industriales canadienses que en 1866, para incitar una subida de la protección aduanera, crearon una asociación, entre otras cosas, para "estallar el espíritu público" y la Canadian Manufacturers' Association que en 1902 creó un Educational Campaign Committee para publicitar sus objetivos. 
caso de nuestro objeto de análisis el objetivo esencial de los movimientos analizados es el de contar con presencia en los medios de comunicación como elementos de difusión de sus demandas y aspiraciones.

b) Campañas apaga-fuegos dirigidas a influir en las decisiones finales sobre las acciones que se ejecutan desde el poder público. Este tipo de campaña intenta secundar las acciones que se realizan sobre otros poderes (legislativo o gubernamental), con una función más de adición que de sustitución. También se pueden concretar como diligencias profilácticas o preventivas sobre actos que se puedan elaborar en el futuro.

Las técnicas utilizadas por las organizaciones que realizan actividades dirigidas a los poderes públicos han sido sistematizadas por Ziegler y Dye (1990: 227-230) en cuatro categorías:

a) Acceso al poder para comunicar las informaciones pertinentes a los decisores públicos.

b) Información. El buen lobbista conoce el proceso legislativo, es experto en el debate político, da información sobre la posición del grupo en diferentes situaciones. Así para poder realizar su trabajo ha de saber utilizar el lenguaje adecuado, conocer a las persones relevantes, saber procesar temáticas diferentes, etc.

c) Movilizar a la base (grass-roots mobilization) a través de cartas, telegramas o llamadas de los ciudadanos hacía las personas pertinentes, siempre bajo la imagen que son espontáneas.

d) Campañas de apoyo de la base a todas las acciones anteriores y sobre todas las cuestiones. Este sostén se realiza con la intención de crear una imagen pública favorable a los grupos o personas o para crear una exigencia pública concreta.

e) En su forma moderna, el juego político se organiza y estructura, de manera creciente, sobre la opinión pública y la contienda política de aspecto simbólico tiende a reducirse significativamente en la batalla por conquistar la opinión. Los grupos de presión intentan apropiarse de las intenciones de esta opinión pública para presentarse ante los poderes públicos como representantes de ésta y defensores de sus pretensiones.

Uno de los puntos esenciales de la dominación política reside en los mecanismos principalmente de orden simbólico, ya que la acción política más importante se muestra de manera subrepticia y solapada y consiste, sobre todo, en la imposición de sistemas de clasificación del mundo. Sobre estas categorizaciones cognitivas, Schumpeter remarcó que toda actividad política necesita acumular un capital simbólico hecho de crédito y confianza que le permita incidir legítimamente sobre las decisiones políticas.

Esta lucha informativa y simbólica se expone como la actuación de numerosos actores políticos, con la pretensión de hacer triunfar su visión acerca de la situación coetánea. Así para Champagne (1990: 83), los estudios elaborados por los especialistas de la ciencia política ya señalaban y remarcaban las estrategias de las minorías acti- 
vas y organizadas (grupos de presión), que pretendían influir sobre las instancias políticas creando, a través de campañas de prensa, principalmente, movimientos de opinión más o menos artificiales.

Los grupos tienen un triple interés para pretender acceder a los medios comunicativos:

1. Obtener que sus reivindicaciones, posiciones, propuestas sean expresadas al mayor número de ciudadanos y que, además, lo sean en términos favorables a sus intereses. Así se obtiene una irradiación social de las demandas grupales, sensibilizando a los individuos acerca de las inquietudes de la organización, que posteriormente se pueden concretar en un aumento de los socios y simpatizantes.

2. Presentar al público y a las autoridades públicas que tienen un respaldo amplio, ya que es frecuente que los responsables de una decisión pública adquieran una impresión de la fuerza de un movimiento, según la resonancia que sus demandas tengan en los mass media.

3. Aparecer como organización que se dedica a una temática determinada, debido a que el proceso de creación del marco de referencia aplicable a un movimiento está profundamente influenciado por el tratamiento que le confieren las informaciones. Esto le permite enmarcar y encuadrar al movimiento y sus objetivos, en una estructura social caracterizada por la multiplicidad de asociaciones voluntarias. En la actualidad, las Organizaciones no Gubernamentales (ONG) tienen, crecientemente, la tendencia a emprender acciones encaminadas a dramatizar lo que son y hacen, con la determinación de exhibirse en el ecosistema comunicativo $^{2}$. Como afirman Molotoch y Lester, para producir la noticia los miembros de los movimientos se han de reunir a una hora inapropiada, en un lugar inapropiado para realizar una actividad inapropiada.

Por lo que respecta a las actividades de las asociaciones ejecutadas con el objetivo de incidir y penetrar en la lógica del sistema de los medios de comunicación, la investigadora Tuchman deja claro que los movimientos sociales deben utilizar la imaginación como instrumento de trabajo, imaginación que comprende desde la dramatización de los acontecimientos hasta la creación de noticias, con la finalidad de aparecer en el sistema comunicativo.

${ }^{2}$ Esta dramatización es una faceta bien captada en el título del estudio de Todd Gitlin (The Whole World is Watching, Berkley, University of California Press, 1980), en el que se resalta que, en el proceso de construcción de la realidad social, los medios de comunicación hacen algo más que observar: dramatizan, crean imágenes vivas y atribuyen el liderazgo de los movimientos. Esta idéntica posición ha sido señalada por Richard Ericson, Patricia Baranek y Janet Chan en Visualizing Deviance: A Studi of News Organization, Toronto, University of Toronto Press, 1987. 


\section{Métodos}

En el presente informe volcamos toda la información hallada en la investigación sobre el movimiento 15-M y Occupy Wall Street. En ambos casos se va a analizar la presencia en medios de comunicación españoles y la actividad de esos movimientos en las redes sociales. Por tanto es un estudio cualitativo y cuantitativo que analiza a través del análisis de contenido la repercusión mediática.

Dados los populares movimientos derivados de las protestas iniciadas durante el 15 de mayo de 2011, con motivo de la indignación ante el sistema actual y las cercanas elecciones autonómicas del 22 de mayo, se procedió a analizar con los parámetros mencionados, una muestra de los ocho periódicos más relevantes del país, a la sazón, El País, El Mundo, ABC, La Vanguardia, El Periódico de Catalunya, la Razón, la Gaceta y Público. Es en ellos donde se buscaron y de donde se extrajeron las noticias para analizar en el presente estudio. Todas esas informaciones han sido objeto de investigación a través de una plantilla de análisis.

También se ha explorado la presencia en las redes sociales sobre los dos movimientos analizados. El análisis embarca el periodo desde 15 de mayo de 2011 hasta 13 de diciembre con unos puntos destacados como la primera semana de las protestas españolas (15 de mayo a 22 de mayo de 2011), protestas internacionales (15 de octubre relacionados principalmente con Occupy a 15 de noviembre). Para examinar el contenido difundido por las principales redes sociales donde está presente el Movimiento $15-\mathrm{M}$, hemos realizado el análisis del contenido y conversaciones circulantes en el muro y streams, mediante una ficha de análisis, además de la aplicación de la herramienta web especializada en seguir las conversaciones y fuentes de ellas (Addictomatic) y otra que especializada en búsqueda y recopilación de menciones sobre el asunto en cuestión mediante varias variables como fuentes, actualizaciones, actitudes (Socialmention).

En el análisis de temática de los mensajes hemos empezado por el análisis del contenido que destaca los temas dominantes en conjunto de las redes y cuentas de Movimiento 15-M, lo apoyamos con el análisis y clasificación debido a aplicación de Socialmention que nos destaca también fuentes principales de estos mensajes. El análisis contribuye también con los tipos de contenido difundido, análisis detallado del DRY (Democracia Real Ya) mediante Addictomatic para destacar los temas más populares y para después demostrar algunos ejemplos de mensajes circulantes que hemos encontrado en varias redes y cuentas a lo largo de investigación.

\section{Resultados}

Efectivamente, el recuento procede a confirmar los resultados vistos en el diagrama: la mayor cantidad de noticias por día empiezan a reflejarse a partir del día 18 y 19 (en que llegan a portada), con un máximo de doce de ellas en El Mundo, 11 en el $A B C$ y 10 en La Vanguardia. Conforme avanzó el tiempo (el presente diagrama sólo llega hasta el día 20 , se llegaron al máximo de noticias entre el 20 y el día 21 , reduciéndose en el día 22 a casi la mitad (imagen 1) 


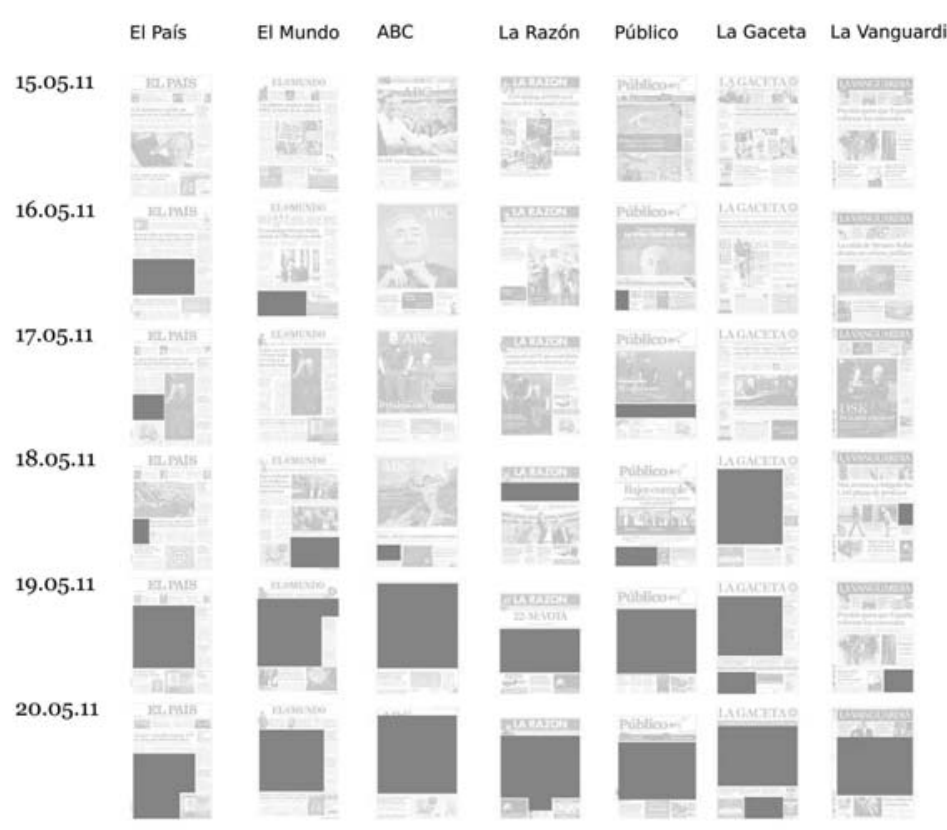

Superficie dedicada al $15 \mathrm{M}$ en las portadas de periódicos

El total de noticias pertinentes compiladas asciende a 323 noticias (se han contabilizado 7 repetidas en varios medios al proceder de agencias, y que por tanto sólo han entrado una vez en cómputo de totales pero se apuntan individualizadas en los medios individuales, lo que elevaría el cómputo a 330).

\subsection{Análisis de los diferentes diarios}

\subsubsection{El País}

En este periódico las noticias reflejadas sobre el movimiento 15-M a lo largo de los días de la muestra (del 15 al 22 de mayo) arroja un total de 39 noticias. Todas ellas de corte informativo, sin reportajes y, si bien con apoyos gráficos (fotografías), con pocos vídeos que sirvieran a nivel testimonial como reportaje o crónica.

Sin embargo el índice bajo de conteo de noticias totales sobre el movimiento 15M (39 en total) mostró porcentualmente más entradas de opinión que El Mundo, con 10 noticias, repartidas de la siguiente manera: 5 de ellas eran cartas al periódico, que mostraban un corte de simpatía hacia el movimiento; 3 eran columnas habituales que se mostraban mucho más críticas con éste, y dos entradas eran de opinión (ningún blog asociado en portada ni de periodista fue reflejado) que mostraban la línea estrictamente legalista y fría del periódico para con el movimiento de los Indignados y 15M, Spanish Revolution, etc, a tenor de las decisiones de la Junta Electoral. 


\subsubsection{El Mundo}

En el periódico El Mundo se sucedieron, a lo largo de los días del 15 al 22, un total de 67 noticias de las cuales 27 eran noticias informativas, 20 artículos de corresponsal o expertos y 7 reportajes más detallados sobre el movimiento, algunos de los cuales (3) se limitaban a ser vídeos colgados en la web y sin comentarios añadidos.

La sección de Opinión de este periódico decidió no alinearse directamente con ni contra el movimiento directamente, utilizando más sutileza mediática al incluir comentarios y opiniones en secciones como "Filosofía". De las tres noticias de opinión encontradas relacionadas directamente con el 15-M, Indignados y Spanish Revolution, proponían una crítica a las acciones tomadas por estas juventudes a la espera de los resultados de las votaciones correspondientes al 22 de mayo. Todo editorial en nombre del periódico se resumía en los vídeos del director Pedro J. Ramírez, colgados en la sección de Opinión y que llevan por título "El Mundo en 2 Minutos". Las 10 opiniones restantes eran de diverso criticismo en la sección de blogs, anidada también en la de Opinión. Son estos blogs, opiniones autorizadas de credencial comprobable y con reputación.

\subsection{3. $A B C$}

El periódico conservador $A B C$ publicó un total de 64 noticias, de las cuales 30 fueron noticias informativas (de Agencia) y 20 artículos con firma. Los reportajes quedaron en 8, y los 6 restantes pertenecen a la sección de opinión. No aparecen blogs en este formato de periódico que reflejen opinión sobre las noticias, implicados directamente en la portada o sección de opinión en el momento de análisis.

Los artículos de opinión son todos editoriales en los que se menciona la situación derivada. El periódico no toma partido directo, sino que utiliza el evento social como mecanismo y muestra de los errores del gobierno socialista, y manifestación abierta de hostilidad a éste por la situación de descontento social.

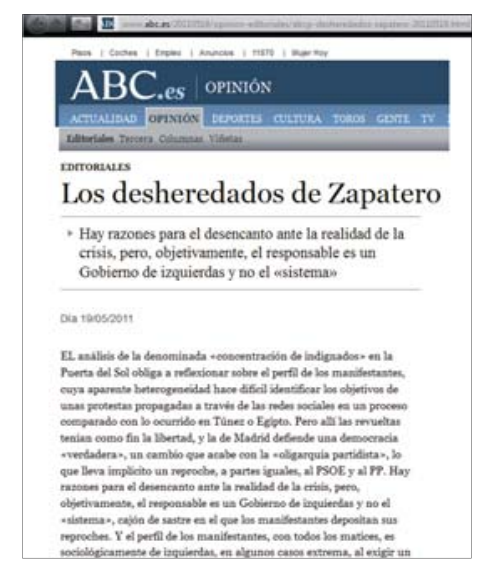

Imagen 2. Ejemplo de artículo de opinión

\subsubsection{La Vanguardia}

Este periódico catalán con un amplio total de 79 noticias publicadas sólo 10 de ellas pertenecen al género de opinión mientras que el resto se reparten en artículos firmados y reportajes.

Los de género de opinión son en su mayoría (7) de blogs asociados a La Vanguardia, mientras que los tres restantes son de artículos de opinión. No existe ningún editorial del periódico durante estos días que mencione, trate, o se centre en los hechos sucesivos en este periodo de tiempo. La línea que siguen en la sección de opinión es de abierta crítica al movimiento $15-\mathrm{M}$, en ocasiones simpatizando en un primer mo- 
mento pero criticando después su falta de ambición política o de fijación de objetivos, la carencia de una unificación en ideología. Se contemplan con escepticismo, esperando que se apoquen al poco tiempo.

\subsubsection{La Razón}

De las 21 noticias publicadas en este medio 17 son noticias informativas, repartidas entre 7 de periodistas firmantes y el resto, 10, de agencias (Agencia EFE, 7); el apartado de Opinión no refleja ningún editorial que mencione directamente y las restantes son artículos o blogs vinculados al medio, muy críticos con el movimiento $15-\mathrm{M}$ y los indignados, así como con Democracia Real Ya.

Y pese a sus duras opiniones sobre el movimiento, prestaron un mínimo indispensable de atención al mismo, centrándose en otros asuntos diarios $\mathrm{y}$ en las elecciones, los partidos políticos y los candidatos antes que en las manifestaciones, que cubrieron, en su mayor parte, por agencias.

\subsubsection{El Periódico}

Muestra un amplio margen de noticias de opinión frente a las informativas. Del total de 26, de las cuales el $50 \%$ se publicaron pasado el día 19 , en que empezaron a ser portada las manifestaciones y concentraciones del 15-M, 10 de ellas son de Opinión (3 editoriales del periódico y diversas columnas de opinión).

Del total restante, 16, 6 de ellas son reportajes de diversa extensión pero nunca menos de una página entera entre las primeras del diario.

Las opiniones editoriales toman partido directamente por los indignados, a quienes alientan desde la tribuna periódica, pero mostrando un corte ligeramente reservado, es decir, dándoles la razón, pero esperando los resultados de los comicios próximos del 22 de mayo, en los que se verificaría o no la efectividad del movimiento indignado. No aparecieron blogs ligados directamente al periódico, sino que la mayoría de las opiniones no editoriales aparecían como artículos firmados por columnistas habituales.

\subsubsection{La Gaceta}

Publicó un total de 34 noticias de las cuales sólo identificó positivamente una como de opinión, y otra como perteneciente a un blog creado puntualmente para dar una visión de la situación desde la óptica crítica de alguien que estuvo en ella. 
La actuación del medio al que pertenece (Intereconomía) no dejó de estar exenta de polémica por una información dada en directo en uno de sus informativos, y posteriormente incluida en el periódico como información, sobre el carácter radical y poco cooperativo con la información periodística que ellos representaban, y a la sazón colgaron en su periódico en edición digital tres vídeos más a tal efecto.

Las informaciones vertidas están calificadas por el medio como noticias informativas, sin aparecer más género como la crónica o el reportaje, si bien dos de las noticias totales estaban incluidas, una en la sección de "negocios" y la otra en "Sociedad".

La mayor parte de los mensajes, en principio, entre los días 15 y 18, eran observaciones sobre los "indignados", y las concentraciones de Sol y Catalunya, y algunos ecos de las concentraciones de Valencia. Los periodistas mostraban una clara hostilidad ante las concentraciones debido a que la mayoría de los manifestantes rechazaban hacer declaraciones públicas, erigir un portavoz o permitir la entrada de periodistas al interior de los campamentos, como reflejan las crónicas escritas a partir del día 19; los periódicos que más animadversión reflejaron con los manifestantes fueron $A B C, E l$ Mundo y sobre todo La Razón y La Gaceta. Ésta última llegó a afirmar en varios de los artículos que "los manifestantes no dejan trabajar a Intereconomía". Entre otras cosas esto se puede percibir en el caso del criticado vídeo del manifestante entrevistado, en el que se aprecian voces que claman que es un montaje, de fondo, y que tuvo mucha repercusión en las Redes Sociales y los medios de Intereconomía (tanto el canal de televisión como el periódico online).

Muchos de los artículos (en torno a los 70, de un total de 323) hablan de conspiraciones tanto de un bando político como de otro, mencionan una supuesta manipulación por parte de intereses poco evidentes ("manos invisibles", es el término literal empleado en los artículos), que propiciaron cuando no instigaron las manifestaciones.

Al avanzar el tiempo llegó el tema de la Junta Electoral. Es el tema que más artículos registra entre los días 19 y 20, haciéndose eco de las declaraciones de la Junta Electoral (declaraba ilegal las concentraciones durante el día de reflexión -el día 21) y las reacciones de los Indignados con respecto a esta prohibición.

Inmediatamente después del día 21, a lo largo del 22 se observó un descenso notorio de la cantidad de noticias, dado que los medios se centraron más en los resultados electorales, aunque sí hubo un ligero incremento el día 23.

Por otro lado, los actores más recurrentes durante estos días en las noticias, fueron la policía, los indignados y los periodistas. Parados y familias aparecen secundariamente, y los vecinos son mencionados, así como los dueños de comercios aledaños a Sol y Catalunya en términos mucho más terciarios. La Junta Electoral aparece únicamente en una fracción de tiempo determinada. Como nombres propios aparecen los alcaldes de Barcelona y Madrid, haciendo declaraciones directas sobre el asunto de los Indignados, siendo recogidas sus declaraciones por los distintos periódicos.

Las secciones en las que aparecen con más frecuencia son Nacional, Política y zonas de opinión como Blogs, Opinión o Editorial. Lo más llamativo del análisis es que en los periódicos de tono más conservador y contrarios a la corriente manifestante, las noticias aparecen en páginas pares de la edición impresa, y en las secciones de Sociedad (cual algo anecdótico) y de Política, entre fuertes críticas de los medios. 
El número de palabras utilizados en los artículos ronda una media de entre $350 \mathrm{y}$ 400 palabras de extensión, y gran cantidad de noticias (el 70\%) tienen soporte gráfico, tanto en edición impresa como digital, así como inclusión de vídeos en éste último formato, en el orden de un escueto $15 \%$, aproximadamente.

Los artículos periodísticos preponderan (75\%) sobre los de opinión, en su mayoría firmados por analistas políticos o periodistas de renombre en los que constan opiniones, en su mayoría duras sobre el tema de los Indignados y las manifestaciones en ambas plazas (Sol y Catalunya), así como los ecos en otras plazas de toda España (Málaga, Valencia, Castellón, Almería, Sevilla, y otras). Estos artículos mencionan como palabras clave y opiniones asuntos relativos a la clase política (protestas contra ella), la plataforma Democracia Real Ya, la Jornada de Reflexión y el tráfico de votos, así como las instigaciones políticas sobre el motivo y la razón de las convocatorias de estas manifestaciones.

\subsection{El tipo del contenido}

En este punto cabe destacar de forma breve que tipo del contenido se pública o más bien utiliza para dar la difusión y repercusión. Empezando por clasificación de los formatos, contamos con la dominancia del formato audiovisual tal como los videos y fotos, logos, posters, fotomontajes, dibujos. Asimismo, se publica el formato textual: varios mensajes y artículos sobre los temas relacionados. Ha de destacar que tanto en publicaciones como en comentarios domina el formato multimedia.

Al cuanto a los géneros periodísticos o más bien relacionados con los varios tipos de comunicación y comunicación 2.0 encontramos: películas, videorreportajes, noticias, comics, avatares, notas de prensa, entrevistas, dossier, artículos, enlaces, tweets, comentarios, foros, entradas en los blogs, informes, eventos en Facebbok, hashtags. Lo que más atención atrae son los videos reportajes sobre las acampadas y protestas y las películas que ganan mucha viralidad similarmente a los tweets (hashtags).

Por último, se puede destacar varios tipos de videos: videorreportajes de manifestaciones, asambleas, acampadas, desalojos y hechos ocurridos, testimonios de los participantes, exposiciones de los "lideres", convocatorias, cortos sobre la historia y resúmenes que incluyen las propuestas (postulados) del movimiento, animaciones con un sentido de humor, además de los videos de crítica, broma, ironía, resúmenes del movimiento, entrevistas y encuestas en la plena calle y dentro de las acampadas en las plazas principales, programas de otros medios, etc. Claramente, los videos con el tema de desalojos y desahucios y los sobre las acampadas en Madrid y Barcelona son más frecuentes.

\subsection{Fuentes de mensajes más utilizados}

\begin{tabular}{|l|c|c|c|}
\hline SOCIALMENTION & Occupy Wall Street & Spanish Revolution & Democracy Now \\
\hline Sources & $\begin{array}{c}\text { Digg Stumbleon Youtube } \\
\text { Picassaweb Facebook }\end{array}$ & $\begin{array}{c}\text { stumbleupon twitter youtube } \\
\text { photobucket facebook ( 25 } \\
\text { mensajes y flickr 20 mensajes) }\end{array}$ & $\begin{array}{c}\text { Twitter photobucket } \\
\text { stumbleupon youtube }\end{array}$ \\
\hline
\end{tabular}




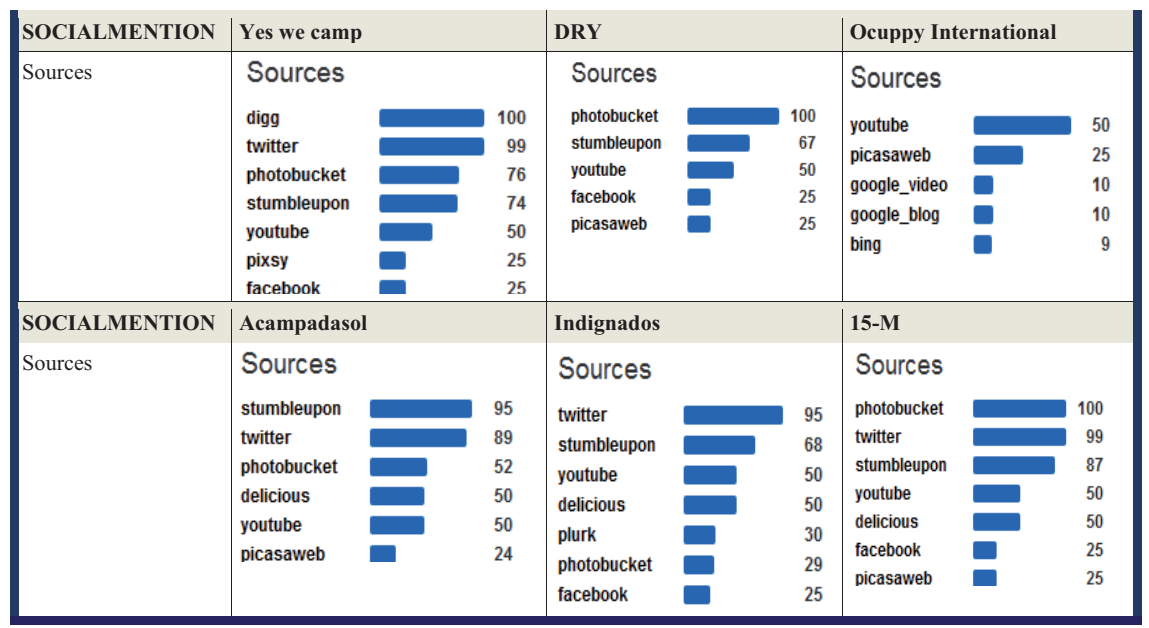

Fig.1. Las fuentes de mensajes. Fuente: elaboración propia sobre bases de datos de Socialmention

Además, hemos comprobado las vías más populares de la difusión de los mensajes, es decir las fuentes más populares de información acerca de Movimiento 15-M. A continuación presentaremos un resumen de las fuentes de mensajes e información sobre 15-M debido a la herramienta de Socialmention (la herramienta que recoge las menciones en social media sobre el asunto que es objeto de la búsqueda e investigación).

Las tres cuentas internacionales como Occuppy, Democracy Now, Yes we camp y Spanish Revolution usan principalmente los marcadores sociales y las redes de contenido audiovisual, después Twitter y al final Facebook, para difundir su contenido y obtener impacto. Similarmente Occupy International que emplea además el blog. La estrategia similar de la sfuentes de conversaciones aplica DRY basándose primero en el contenido de las fotos en varios sitios sociales online, después de marcadores sociales y contenido de video y ya después mediante la gestión de la comunidad. Por lo cual el mayor peso de su viralidad es debido al contenido circulante y no comunidades. A pesar del uso extensivo de Twitter, el programa Socialmention no destaca esta red dentro de las fuentes de conversaciones utilizadas por DRY.

En cuanto al análisis de Socialmention, mientras Acampadasol, Indignados y 15M usan primero los marcadores sociales, las varias redes y sitios del contenido audiovisual y Twitter como fuentes principales de la difusión del contenido y fuentes de las conversaciones. Por lo cual podemos resumir, que gracias al uso de dichas tácticas, esto explica su gran visibilidad en los medios sociales y el poder en esta arena de comunicación 2.0. Gracias a esta estrategia mixta de las redes del contenido audiovisual de varios formatos ( tanto fotos como videos) y potenciada por aportar el contenido que por su relevancia y ser atractivo ocupa destacadas posiciones en las marcadore sociales, potenciada por el potencial de viralidad de Twitter se obtiene mayor presencia en Social Media ya que estas redes tienen mayor potencial en difusión de las ideas sociales, protestas y asi ayudan obtener la mayor repercusión de una 
protesta en el nivel nacional e internacional. Contenido audiovisual es más atractivo por lo cual atrae atención, los marcadores "marcan" temas de gran interés del dicho momento ${ }^{3}$. Además, Indignados y 15-M se apoyan por Facebook para fomentar sus comunidades.

La gestión de comunidad junto con la presencia en las plataformas sociales del contenido ayudan a obtener más poder, mientras que definitivamente marcadores sociales, Twitter y después el contenido audiovisual atractivo y relevante ayuda al ganar impacto expresado por el alcance y visibilidad, es decir mayor publicity en las redes sociales online. Comunidad es significativa para poder ejecutar influencia social online, mientras que los sitios sociales de conversaciones fomentan viralidad que potencia presencia en Social Media. El elemento común e imprescindible como herramienta de difusión de las protestas sociales y sus ideas o sentimientos junto a las soluciones es en la era de Social Media el contenido audiovisual. Gracias a todo tipo de los formatos de este contenido ya sean fotos o video la gente se vinculan al movimiento, esto despierta su interés en el tema de protestas, obtiene repercusión nacional e internacional y al final involucra la comunidad en las conversaciones ya que sabemos que un imagen vale más que mil palabras. Estas tácticas son importantes factores de gestión efectiva y eficaz del movimiento social como en el caso de 15-M y justifican su importancia en estrategia general de Social Media.

\subsection{El contenido del perfil: mensajes}

Para examinar el contenido de los perfiles hemos realizado una observación no participante de las diversas cuentas relacionadas con 15-M, sobre todo en Facebook y Twitter que son principales canales de información, pero además de Youtube y Flickr, por dominancia del contenido audiovisual que recogen y difunden estos dos servicios sociales.

Idiomas dominantes de mensajes son el español e inglés de los perfiles. Además en caso de las cuentas internacionales como Democracy Now y Occupy International menciones y publicaciones son en varios idiomas, incluyendo las idiomas asiática y árabe. El uso de múltiples formatos formales y comunicativos además las cuentas redactadas de manera multilingüística potencia el alcance e impacto de las comunicaciones.

Uno de los eventos con mayor importancia cubierto por las conversaciones en las redes sociales teniendo el mayor impacto era 15 de Octubre relacionado con los temas de revolución global y democracia.

Ejemplos de las temas publicadas en Facebook a lo largo de siete meses de la duración del Movimiento 15-M y nuestra investigación, en este caso publicado por una de las cuentas con mayor actividad en publicaciones (mayor numero de las publicaciones combinado con mayor frecuencia de los posts):

${ }^{3}$ Lo que también explica la falta de la involucración en conversaciones de las comunidades de Acampadasol, Indignados o 15-M.Siendo los perfiles mayoristas del movimiento y creados más al corriente de la protesta al aparecer se basan en los temas populares de cada momento particular que dejan ser relevantes más tarde. 


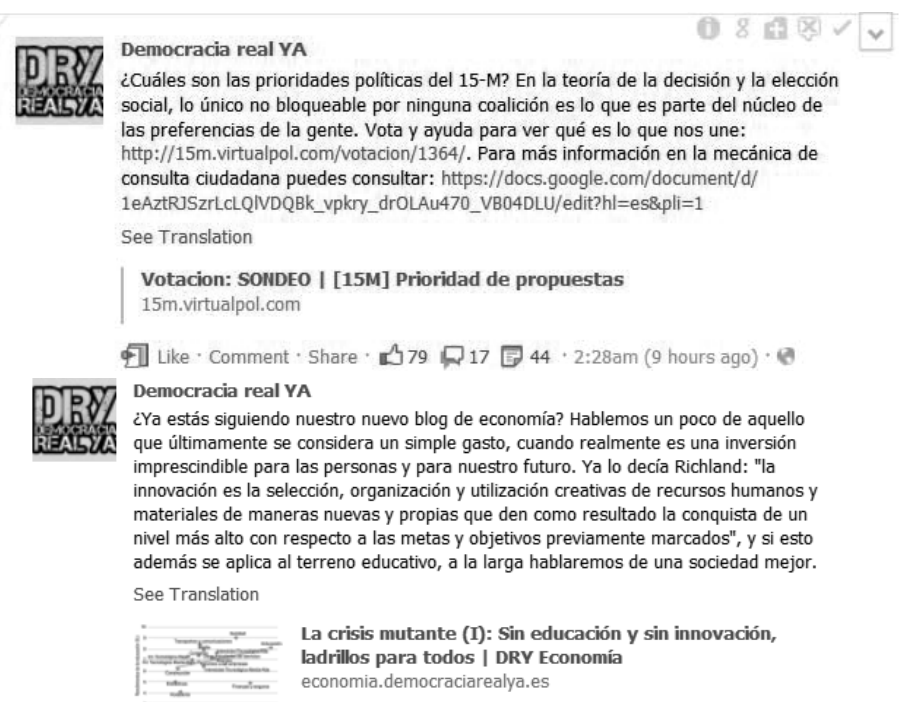

Imagen 5. Ejemplos de las publicaciones de Democracia real Ya en Facebook. Fuente: DRY en Facebook

Estos ejemplos nos enseñan claramente las primeras ocupaciones del movimiento social español. Una es dar la voz al público para que seleccione las temas de importancia para él, las prioridades que en este caso son las elecciones sociales y la importancia de influencia de la decisión ciudadana que según el movimiento no está lo suficiente ejercida en la democracia actual. El segundo además de la preocupación por la política es la preocupación por la economía. En este caso se ha abierto un sitio para el discurso sobre la crisis económica y las propuestas de soluciones para ello.

Primero, llama la atención el uso de la red social por el grupo para hacer las consultas sociales con el resto de los participantes y fans del movimiento y de la cuenta. Aquí encontramos la doble ventaja: la red social une los simpáticos de la causa social y es un espacio del discurso más abierto e proporcionando la voz y la igualdad. Además puede ser una plataforma de las consultas sociales e investigación de la opinión pública por las cosas de interés social, que en tiempos antes de la era de social media demandaba muchos recursos para hacer encuesta de esto tipo. Además, red social y las varias plataformas sirven para diferentes objetivos: difusión, promoción, viralidad, análisis, discusiones e investigaciones de la opinión pública. En la segunda publicación vemos que se ha creado un blog para hablar de economía actual ya que el blog parece una herramienta perfecta para las conversaciones y análisis acerca de este tema. Asimismo, se ha utilizada Facebook para dar más difusión y visibilidad al blog.

En resumen, DRY se involucra mucho en crear de las redes sociales unas plataformas de las conversaciones sobre las propuestas concretas como mejorar la democracia y economía actual. Los temas estos ocupan mucho espacio dentro de la temática de total de las publicaciones. Además, la cuenta sirve para las convocatorias de concentraciones en plazas centrales de las ciudades españolas, tal como lo ilustra la imagen 6: 


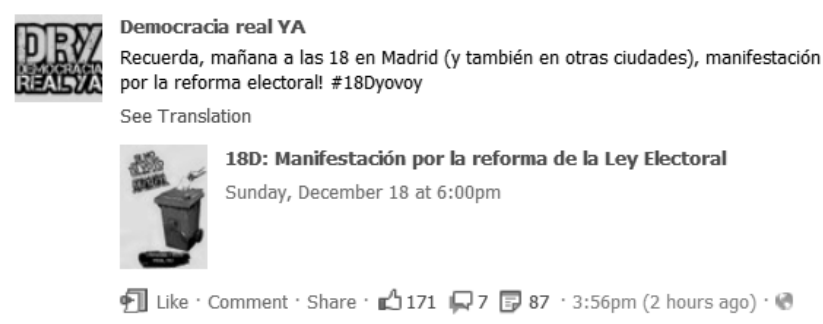

Imagen 6. Publicación convocando la manifestación. Fuente. Facebook

Ejemplo de la convocatoria de manifestación son las publicaciones que mantienen vivo el movimiento en el mundo offline, es decir, señalan su significado y la presencia por seguir concentrando la gente en las protestas en la calle por diversas causas, entre ellas las más destacadas son acerca del tema del ley electoral y los desahucios. A continuación se encuentra el mensaje destacado derivado de Twitter, cuando se nombró el indignado el personaje del año:

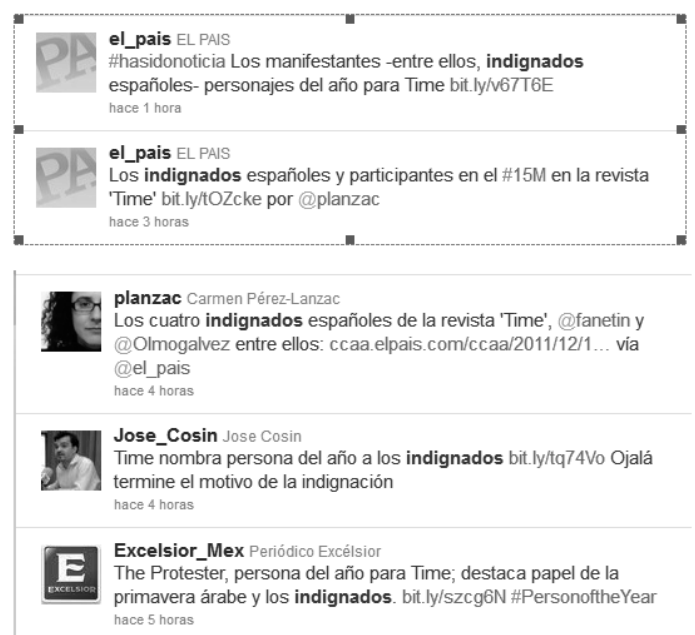

Imagen 7. Evento de nombrar Indignado de la persona del año por Times.Twitter. Fuente: Twitter.

En la imagen 4 encontramos el ejemplo de las publicaciones en Twitter sobre el hecho de elegir por Times el protestante español- The Protester o más bien el indignado- al personaje del año 2011. No solo aparecieron los comentarios de los bloggers, participantes del movimiento, cuentas del movimiento, las personas privadas que apoyan la idea, sino fue ampliamente comentado y difundido por los periódicos españoles mediante sus cuentas en social media. Este hecho tuvo una gran difusión el día de la noticia y su seguimiento en social media las días siguientes. Era cualificado por las herramientas de medición com Addictomatic como top y hot topic en todo tipo de la cuentas en Facebook y Twitter sobre todo. La repercusión era muy positiva ya que no hemos encontrado los comentarios negativos sino positivos y destacando el orgullo del poder de los ciudadanos españoles que obtuvieron tan gran reconocimiento internacional de sus esfuerzos y repercusión de su indignación. 
Debido al desarrollo de las redes sociales, as protestas sociales ganan más poder, importancia e influencia política incluso en el nivel internacional. Para ello no solo que gana su apoyo por parte de la influenciadores del mundo social online o los demás usuarios y los medios tradicionales presentes en las redes sino también porque los lideres o participantes activos de este movimiento que lleven las cuentas del movimiento en social media de forma de Community Mamanger y en muchos casos profesionalmente. De este modo, el movimiento de mantenga presente y vivo a lo largo del tiempo en realidad online asi como offline.

En la imagen 5 presentamos el ejemplo de la publicación por el aniversario del movimiento 15-M que nos enseña de forma multimedia, interactiva y concisa el significado y el desarrollo del movimiento de modo resumen. Pero ilustra también la repercusión y sobrevivencia de esta protesta social en social media que se ha llevado a cabo gracias a la implementación adecuada de las herramientas de social media. Vemos que la publicación en solo 5 horas ha conseguido casi 880 me gusta y 170 la han compartido mientras que 73 la han comentado, en mayoría demostrando su apoyo, lo que ilustra la popularidad del tema y de la idea y su influencia incluso siete meses después de las más concentraciones de $15-\mathrm{M}$, al contrario de los medios tradicionales que ya a estas fechas no publican mucho sobre el tema.

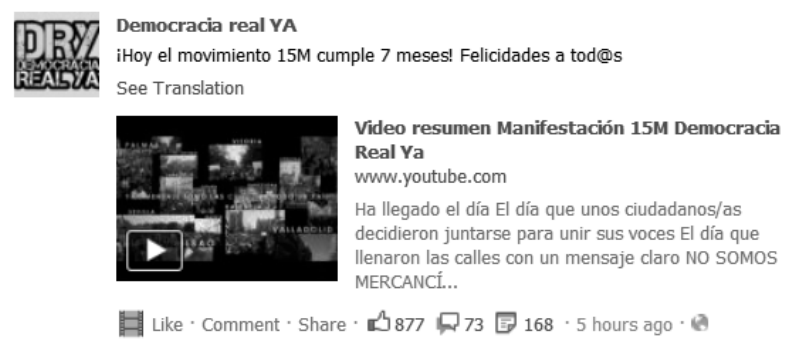

Imagen 8. Ejemplo de la celebración de aniversario de movimiento 15-M y su desarrollo en Facebook. Fuente: Facebook

Lo más importante es que la final de la cuentas la protesta civil no es el cosa que divide la sociedad sino la integra incluso más siendo un signo de despertar o mejora de la sociedad civil. Las redes sirven aquí como herramientas de organizarse, difundir la información, elaborar las nuevas ideas y expresarse por la gente que han sido excluido del discurso principal en los medios grandes o incluso han sido criticados por la participación en aquello.

\subsection{Occupy Wall Street}

Tras los resultados del 15-M, juzgados por la prensa como ineficaces (las asambleas y comunicados pedían voto nulo, con una gran profusión en las redes) por los resultados obtenidos en las elecciones generales españolas, independientemente a ellas y debido a la crisis mundial económica, generalizada, en los Estados Unidos apareció el movimiento Occupy Wall Street. Al contrario que el movimiento Democracia Real Ya y de los "indignados", se centró de inmediato (sin que mediaran elecciones, como en el caso español) en reivindicaciones y críticas feroces contra el sistema político y 
económico de su país. Las asambleas más importantes, sitas en Nueva York y Los Ángeles se centraron de inmediato en asentarse en un campamento y crear protestas y consignas. De inmediato reaccionaron los medios políticos, las respectivas alcaldías, centrándose sobre todo en la de Bloomberg, la de Nueva york, que apoyó tácitamente a los manifestantes en tanto que tenían derecho a hacer esas manifestaciones, y a tenor de las resoluciones del caso español, se aprestaron a pasar el tiempo lo mejor posible, que le llevara el acontecimiento.

El caso de Occupy Wall Street refleja un notable número menor de incidencia e importancia en los medios españoles con un total de 98 noticias en desde el inicio del movimiento hasta la fecha actual en que se escriben estas líneas, frente a las 323 encontradas en el caso anterior en el plazo de una semana.

El movimiento popular iniciado en Estados Unidos y parte de Europa, posterior al conocido como Spanish Revolution tuvo un impacto mucho menor en los medios españoles con menos de un tercio de las noticias que se consiguieron ante el evento nacional, quedando el total de aquellas en 98 entre los mismos periódicos consultados para el primer caso.

Del total de 98 noticias, la mayoría se agrupan principalmente en noticias informativas, dejando apenas 5 en el apartado de Opinión, en forma de artículos firmados (2) y editoriales (3) en distintos periódicos (gráfico 1).

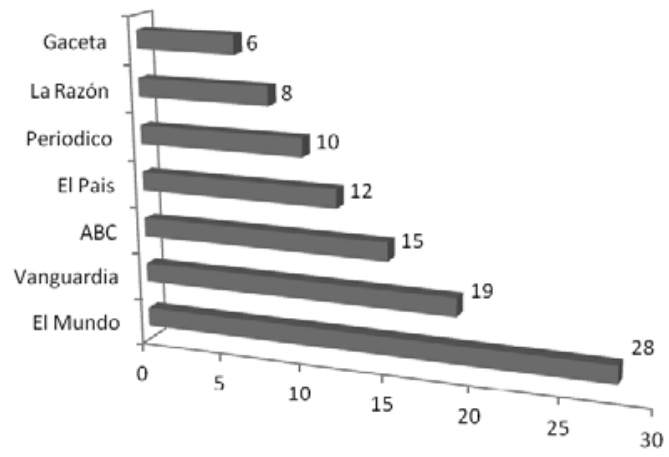

Gráfico 1. Número de noticias por diarios. Fuente: elaboración propia

Sin embargo lo más debemos destacar son el género de entrevista a expertos de diversos campos que se incluyen en algunos periódicos consultados, que se incluyen en los reportajes, 12 en total. En cinco de estos se incluyen entrevistas con profesores de economía y otras disciplinas como ciencias políticas (1) o Sociología (2).

El periódico que recogió más reportajes fueron El Mundo y La Vanguardia, con tres cada uno. Si bien el género del reportaje no se explotó mucho más pese a lo lejano de los lugares de la noticia, éstas fueron reforzadas en gran medida con varios vídeos alojados en diversas plataformas de Internet.

Siguiendo la metodología del caso anterior encontramos que la mayoría de las noticias recaen en la sección de Internacional de los periódicos, así como en menor medida en las de economía y secciones de entrevistas directas y opinión.

Estas opiniones informadas son, en su mayoría, de profesores de universidad, políticos, miembros de la comunidad y manifestantes que, con respecto al caso español, sí tenían mensajes directos que comunicar a los medios. Los profesores universitarios, como opiniones informados, son: profesores de Económicas de la Universidad de Nueva York, catedrático de Economía, Profesores de política y de Sociología. Los políticos en su mayoría están ligados al ayuntamiento y trasladaban la opinión de éste con respecto a los manifestantes. 
La extensión media de los artículos encontrados es de 360 palabras y un 65,5\% tienen soporte gráfico, y en algunos casos (13) vídeos alojados en la misma página que la noticia.

Los términos más utilizados aparte del consabido "Indignados" que comparten con el caso español, se relaciona con los sucesos subsiguientes a la ocupación por tres semanas en Nueva York: la actuación policial, que en nuestro estudio previo del caso 15M quedó fuera al limitar el tiempo a la semana de las elecciones. La actuación de los antidisturbios ocupa la terminología más recurrida: policía, disturbios, protesta, violencia, gas pimienta, muertes, arrestos, agresión policial y cargas policiales.

Los actores más mencionados y que intervienen en las noticias están presididos por la figura del Alcalde de Nueva York, Bloomberg, y sus numerosas declaraciones en los medios. La alcaldía de Los Ángeles no llega a tener tanta notoriedad, además de hacer una declaraciones mucho más favorables, aunque las cargas policiales en la Universidad de California llegaron a ser notorias en noticias posteriores e incluso rozaron el "meme", esto es, convertirse en un recurso expresivo en el ciberespacio, con las imágenes de uno de los policías rociando indiscriminadamente a los manifestantes sentados en el suelo. Incluso llegó a saltar a los medios las llamadas a la acción hacia el presidente Obama, aunque no acabaron de plasmarse en respuestas de éste hacia la situación que se ha venido gestionando como un evento local y federal, pero no nacional (en la terminología de los USA).

Por lo tanto, las noticias más habituales eran sobre la temática de las detenciones, desalojos, arrestos y protestas, antes que sobre las intenciones de los manifestantes. Aunque sí hay una identificación más notoria con respecto a un movimiento cohesionado, como el Occupy.

Por otro lado se ha publicado también por el propio Facebook, la lista de los temas dominantes incluye Occupy Wall Street. Así, hemos encontrado un ranking publicado por U.S. Politics on Facebook que ha analizado los temas más populares o sea hablados en Facebook en 2011: Top Facebook Political Articles

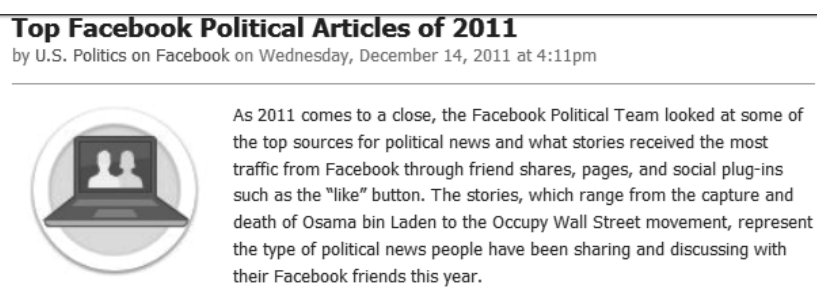

1. Daily Kos: Open Letter to that $53 \%$ Guy

2. Washington Post: Obama's and Bush's effects on the deficit in one graph

3. FOX News: Should U.S. Get Involved in Syria?

4. CNN: Osama bin Laden, the face of terror, killed in Pakistan

5. Salon: "USA! USA!" is the wrong response

6. Huffington Post: Michelle Obama Dances 'The Dougie' \& 'The Running Man'

7. Huffington Post: Obama's Birth Certificate Through The Eyes of A Birther

8. CNN: Think Occupy Wall St. is a phase? You don't get it

9. Huffington Post: Sarah Palin's PAC Puts Gun Sights On Democrats She's Targeting In 2010

10: MSNBC: Anti-gay marriage group fakes support with doctored photos

11. Huffington Post: Conservative Pie: Republicans Introduce Legislation Redefining Pi

12. Huffington Post: Westboro To Picket Funerals of Arizona Shooting Victims

13. MSNBC: Web's bin Laden 'death photo' (just the photo) is fake

14. LA Times: Michele Bachmann is worried about the Renaissance

Fig. 2. Temas políticas más hablados en Facebook. Fuente: Facebook of 2011 .

Entre ellos observamos que el tema de OCCUPY Wall Street ocupa las posiciones de $8,17,20,22,31,33,39$ y 40 en los 50 destacados. Además, lo que llama nuestra atención es no solo hecho que domina las posiciones dominantes en el ranking o se en- 
cuentra en la cabeza de ello sino que es tema que domina entre las 50 destacados lo que podemos resumir en la siguiente manera- los artículos y conversaciones sobre Ocuppy Wall Street dominan el discurso político en Facebook en 2011, ya sea por su relación directa o indirecta con el movimiento de las protestas sociales en Estados Unidos (se encuentra por ejemplo un tema que la clase media apoya las protestas o el tema sobre impuestos para los más ricos).

\section{Conclusión}

Los medios trataron como una noticia internacional y vagamente inspirada en los acontecimientos vividos en España en el 15-M. Pero el protagonismo casi absoluto vino dado por los eventos violentos, las cargas policiales y las repercusiones de las manifestaciones. Todo ello como noticias internacionales sin apenas lazos con España ni los Indignados, pero sí se utilizó la misma terminología.

El 15-M quedó reflejado en los medios de prensa digital y convencional tardío como evento popular y poco analizado por los medios.

Los medios reflejaron información parcial y de cauces externos. Hubo muy poca información emanada o colectada del interior del "Movimiento 15-M".

Las noticias más relacionadas y extendidas sobre los acontecimientos en torno a los días 15 y 22 de mayo son aquellas en las que aparecían manifestaciones del gobierno central y la Junta Electoral.

Hubo un descenso de más del 50\% de las noticias a partir del día 22 para centrarse en los resultados electorales, y dejando de lado el movimiento 15-M, juzgándolo como ineficaz.

El surgimiento de las redes sociales han cambiado de manera de protestar en la que las protestas reales en el mundo offline, que nutren y mantienen el nivel de soporte y del energía necesaria para que las protestas alcancen sus objetivos en el mundo online- creando un círculo de alimentación continua y mutua. Asimismo, la parte de sociedad que está protestando se independiza de los medios de mainstream mientras que social media sirven para continuar las protestas de forma simultánea en dos realidades. La continuación de las protestas tiene sus picos y bajos de las conversaciones o soporte y de la actividad pero en general crea una línea estable del espíritu de sociedad y protesta civil.

Los Social Media se muestran como una herramienta poderosa en creación, gestión y difusión de una protesta social. Además, podemos ver que en su mayoría el público objetivo del movimiento se encuentra en Facebook y Twitter y los demás redes sociales lo que nos lleva a la conclusión que la nueva poder pertenece a un ciudadano digital, ciudadanía digital y allí nos encontramos con la sociedad civil verdadera. Es la sociedad civil digital que no solo está familiarizada con las TIC ganando más poder en expresión de su indignación pero es capaz transferir su indignación y mantener la repercusión de ella en ambos mundos offline y online. Es también el hecho que puede servir para demostrar que no es válida la conclusión que la sociedad de internautas es vaga o no les interesa la política y no sea capaz organizarse.

Todo esto nos demuestra una poder doble: por un lado de los medios sociales online en difusión de las causas e ideas sociales mediante los mensajes atractivos, inte- 
resantes y relevantes y como una herramienta independiente y eficaz en gestionar las protestas sociales en el nivel internacional. Por otro lado nos enseña el poder de los movimientos sociales y su rapidez en desarrollo y difusión, así como capacidad de conseguir apoyo internacional y poder del nuevo usuario socialmente consciente y hiperconectado que puede cambiar el mundo con los demás ciudadanos por simplemente hecho de facilidad de vinculación, contacto mutuo y enfoque participativo. Con los movimientos analizados se constata que los ciudadanos organizados pueden combinar las estrategias de comunicación para expresarse, unirse, movilizarse en el ámbito comunicativo offline y online.

\section{Referencias bibliográficas}

TOCQUEVILLE, Alexis de (1980): La democracia en América. Madrid, Alianza.

MILBRATH, Lester (1963): The Washington Lobbyist. Chicago, Rand McNally.

FINER, Samuel (1966): El imperio anónimo. Un estudio del "Lobby" en Gran Bretaña, Traducción y estudio previo de Juan Ferrando Badia. Madrid, Tecnos.

ZIEGLER, Harmon y DYE, Thomas R. (1990): The irony of Democracy. An Uncommon Introduction to American Politics. Belmont, Cole Publishing Company, ( $8^{\mathrm{a}}$ edición).

CHAMPAGNE, Patrick (1990): Faire l'opinion. Le nouveau jeu politique. Paris, Les Éditions de Minuit.

TUCHMAN, Gaye (1983): La producción de la noticia, Estudio sobre la construcción de la realidad. Barcelona, Gustavo Gili.

MOLOTOCH, Harvey L. y LESTER, Marylin (1974): "News as purposive behavior. On the Strategic Use of Routine Events, Accidents, and Scandals", en American Sociological Review, nº 39, Feb. 1974, pp. 101-112. 